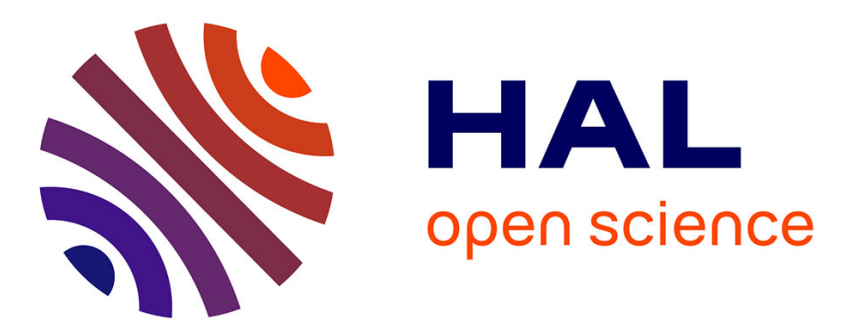

\title{
Les dimensions spatiales des relations familiales en migration : les enjeux autour de la grossesse en migration
}

\author{
Anne-Cécile Hoyez, Clelia Gasquet
}

\section{To cite this version:}

Anne-Cécile Hoyez, Clelia Gasquet. Les dimensions spatiales des relations familiales en migration : les enjeux autour de la grossesse en migration. Norois, 2020, 257, pp.7-20. 10.4000/norois.10448 . halshs-03432317

\section{HAL Id: halshs-03432317 \\ https://shs.hal.science/halshs-03432317}

Submitted on 17 Nov 2021

HAL is a multi-disciplinary open access archive for the deposit and dissemination of scientific research documents, whether they are published or not. The documents may come from teaching and research institutions in France or abroad, or from public or private research centers.
L'archive ouverte pluridisciplinaire HAL, est destinée au dépôt et à la diffusion de documents scientifiques de niveau recherche, publiés ou non, émanant des établissements d'enseignement et de recherche français ou étrangers, des laboratoires publics ou privés. 


\section{Les dimensions spatiales des relations familiales en migration : les enjeux autour de la grossesse en migration.}

\section{Auteures :}

Clélia Gasquet-Blanchard ${ }^{\mathrm{a} *}$, Anne-Cécile Hoyez ${ }^{\mathrm{b}}$

a Maîtresse de Conférences en Géographie, UMR 6590 ESO, Laboratoire Rennes 2, Département SHS, EHESP, 20 avenue George Sand, 93210 La Plaine St Denis, Ecole des Hautes Etudes en Santé Publique (clelia.gasquet@ehesp.fr )

${ }^{\mathrm{b}}$ Anne-Cécile Hoyez, Chargée de Recherche CNRS UMR ESO/Université Rennes 2 Bureau N306, Université Rennes 2 ; Maison de la Recherche en Sciences Sociales Laboratoire ESO Rennes - UMR 6590 ESO CNRS Place du Recteur Henri Le Moal, 35043 RENNES Cedex, (anne-cecile.hoyez@univ-rennes2.fr)

\section{Résumé :}

Cet article repose sur l'analyse d'observations et d'entretiens conduits dans le cadre de plusieurs terrains de recherches. Il propose une réflexion de fond sur les inégalités en santé périnatale, notamment en termes d'accès aux soins pour les publics primo-arrivants. Ces terrains ont participé à structurer une réflexion autour de la grossesse, et nous conduisent, dans cet article, à aborder les dimensions spatiales identifiées dans les dynamiques relationnelles, et notamment familiales, pour les femmes migrantes récemment arrivées en France et vivant un épisode de grossesse. Nous montrons que les enjeux existant pour ces femmes impliquent des trajectoires tantôt d'éloignement, tantôt de rapprochement avec leur famille plus ou moins élargie. Ces logiques impliquent d'apprendre à gérer la distance, notamment pour le père, et de prendre en considération des contraintes sociales et institutionnelles qui ont un impact important et durable dans la vie maritale. Enfin, ces dynamiques mettent également en avant qu'elles peuvent être guidées par des relations affinitaires décidées par les femmes.

\section{Summary:}


This article focuses on a synthesis analysis of observations and interviews conducted in the framework of several research fields. It provides an in-depth reflection on perinatal health inequalities, particularly in regard to access to healthcare for newly-arrived immigrants. These projects have contributed to structuring a reflection on pregnancy. In this article, we propose to address the spatial dimensions identified in the relational dynamics, and in particular family dynamics, for migrant women who have recently arrived in France with a pregnancy. We show that the challenges for these women involve trajectories that are sometimes distant and sometimes closer to their more or less close family. These logics require learning to manage distance, especially for the father, and to take into consideration social and institutional obstacles that have a significant and lasting impact on marital life. Finally, these dynamics also highlight the fact that they may be guided by affinity relationships decided by women.

Mots clés : trajectoires, périnatalité, migration, grossesse, famille, pratiques spatiales

Key words: trajectories, perinatality, migration, pregnancy, family, spatial mobilities

\section{INTRODUCTION :}

Dans la continuité des travaux portant sur les migrations des femmes, nous cherchons à rendre visible un ensemble de facteurs qui entrent en jeu dans la coordination des parcours de grossesse en France. Les propos se situent à l'interface entre les objectifs de l'action publique (santé publique et domaine sanitaire et social) et les expériences vécues par les femmes dans les mondes sociaux où elles évoluent. Ce faisant, l'article propose de déconstruire un ensemble d'idées reçues sur les statuts cumulés de «femme/enceinte/primo-arrivante », qui peuvent conduire à un vaste champ de stéréotypes et de représentations négatives ou réductrices (femmes inactives, venues suivre un conjoint, etc.), notamment décrites et critiquées par plusieurs chercheuses (Kofman, 2004; Catarino, Morokvasic, 2005; Morokvasic, 2008; Schmoll, 2014; Gerbier-Aublanc, 2017). Dans cet article, sont particulièrement éclairées les situations de précarité et d'éloignement-isolement vécues par ces femmes. En effet, on observe d'une part la surreprésentation des femmes primo-arrivantes dans les lieux de soins ou d'accueil dédiés aux publics précaires (Rietch 2014; Médecins du Monde, 2020 ; Gasquet-Blanchard, 2020). Si considérer la précarité comme unique prisme par lequel doivent être abordées les trajectoires des femmes migrantes enceintes apparaît comme réductionniste (Kofman, 2004), elle constitue néanmoins une partie importante des réalités rencontrées en France par les femmes interviewées au cours de nos terrains de 
recherche (voir méthodologie) ${ }^{1}$ (Gasquet-Blanchard, Hoyez, 2015). D'autre part, l'isolement de ces femmes, même s'il n'est pas l'apanage des personnes en situation de précarité (et inversement), se donne à voir à travers une dialectique d'éloignement-rapprochement. L'éloignement spatial avec les proches restés au pays induit un rapprochement avec des compatriotes dans les espaces du quotidien en France, participant au tissage de nouvelles sociabilités, voire de nouvelles implications familiales (Guerraoui, Strum, 2012 ; Battaglini et al., 2002).

Mais l'éloignement social et la précarité, quand ils se conjuguent, sont souvent associés aux risques d'isolement pour l'action publique. Par exemple, on constate la prépondérance de dispositifs à destination des femmes (enceintes ou non) seules, ou des familles monoparentales (Neyrand, 2015). Cette organisation porte à faire perdre de vue la présence, parfois immédiate et à proximité, parfois plus distante, des conjoints et pères, des familles et des nouvelles sociabilités créées au cours de la trajectoire migratoire, révélées notamment dans les approches biographiques (Collet, Veith, 2013). Par ailleurs, la migration peut participer à des situations d'errance (sans pour autant définir une relation de cause à effet). Claudio Bolzman (2014), décrit des situations « où des personnes en exil sont amenées à vivre des situations d'errance, et à se trouver ainsi dans des dispositifs sociaux qui n'étaient pas, en principe, prévus pour elles» et montre comment «le déplacement indéfini ou provisoire, dans un temps plus ou moins continu, sur un ou plusieurs territoires » implique une errance sur le plan administratif (marquée par les variations des titres de séjour, statuts, types de protections juridiques, ouvertures des droits sociaux, déclaration du nouveau-né) qui entravent la possibilité des individus à être et se sentir légitime dans un lieu, y résider, y vivre. Dans ces situations d'errance, les femmes enceintes en migration nécessitent donc un soutien, dans la sphère sociale et institutionnelle. Battaglini et ses collègues (2002) soulignent que le rôle de soutien est généralement assuré par les travailleurs sociaux et/ou les proches, notamment les conjoints dans un cadre de «réagencement du genre à la faveur de la migration » (Guerry, Thébaud, 2020) et qui activent des aides de nature instrumentale, matérielle, morale ou informative souvent empreintes de normativité (Planche, 2014). Pourtant, il arrive que ces soutiens à destination des femmes soient insuffisants.

\footnotetext{
${ }^{1}$ De plus, analyser la précarité ne doit pas empêcher de rendre compte de la situation de femmes moins précaires mais fragilisées dans d'autres domaines, comme par exemple : embauche dans le champ professionnel à des postes sous qualifiés au regard de leur expérience, assignations aux métiers du care et du domestique sous qualifiés et sous rémunérés, renoncement au sein des ménages aux carrières professionnelles féminines au profit des carrières masculines notamment, comme l'ont montré les travaux de Karine Duplan (2016).
} 
Aussi, nous nous intéresserons ici surtout aux sociabilités des femmes enceintes primoarrivantes dans leurs espaces du quotidien et moins à leur état de santé. En effet, migration et grossesse se présentent comme deux événements biographiques, mais aussi deux « expériences fondatrices» (perte et refonte d'un réseau social ailleurs ou à distance, accolées à un changement de statut familial) particulièrement situées dans des contextes qui complexifient ces expériences. Dès lors, nous souhaitons, dans cet article, éclairer la dimension spatiale de la grossesse en situation de migration, notamment autour des dialectiques entre, rapprochement/éloignement ; mobilités/distances, sociabilités/spatialités, et ce en contexte urbain et au quotidien, particulièrement concernant l'hébergement et l'environnement social et relationnel de ces femmes.

Nous proposons de traiter, dans une première partie, de la grossesse comme événement social dans le contexte de la migration et d'interroger comment s'articulent les dimensions spatiales, sociales, administratives et temporelles des trajectoires. La deuxième partie met l'accent sur la situation des couples quand la grossesse se déroule en migration : la place des pères, peu visibilisée dans les recherches, alterne entre présence, absence et/ou tenue à distance de certains dispositifs d'aide, ce qui oriente fortement les expériences de leurs compagnes enceintes. Enfin, de façon transversale, dans une troisième partie, nous nous intéressons à la façon dont la vie sociale se trouve ré-agencée dans un contexte de grossesse en France, notamment dans les différentes façons de s'investir pour les proches et dans la présence ou la séparation d'avec les enfants aînés. 


\section{Méthodologie :}

Cet article se base sur un corpus d'entretiens issus de différents programmes de recherche s'intéressant aux trajectoires de femmes enceintes : celles de femmes enceintes ayant accouché d'un enfant prématuré (réalisés dans le projet DISPARITE ${ }^{2}$ entre 2012 et 2014 menés dans 4 villes françaises, au sein de 8 services de néonatologie et en maternité). Au sein de cette recherche, nous avons sélectionné les 15 entretiens réalisés auprès de femmes arrivées depuis moins d'un an sur le territoire (sur les 120 au total). Cette étude nous a amenées à approfondir le lien entre grossesse, migration et accès aux droits, notamment l'accès aux soins (intégrant de fait une diversité de statuts administratifs au sein du public rencontré). C'est ce que nous avons également investigué, dans le cadre du projet MIGSAN ${ }^{3}$, avec la réalisation de 29 entretiens réalisés entre 2013 et 2017 auprès de femmes primo-arrivantes enceintes à la consultation et à la maternité d'un CHU (l'ensemble de ces entretiens sont ici mobilisés). Par ailleurs, dans le cadre d'un partenariat institutionnel et conventionné avec un réseau médico-social francilien de prise en charge des femmes enceintes en situation de grande précarité, nous menons une approche de long terme et réalisons régulièrement depuis 2013 des observations et entretiens avec les femmes incluses dans ce réseau de santé. La majorité des femmes suivies au sein de ce réseau ont récemment migré sur le territoire.

$\mathrm{Au}$ total, ce sont 44 entretiens semi-biographiques qui sont mobilisés dans cet article. La méthodologie de l'ensemble de ces projets se base sur une approche qualitative, à partir d'entretiens conçus pour appréhender les trajectoires de la grossesse et de la migration, le parcours de soins ainsi que les éléments contextuels de ces trajectoires (statuts administratifs, socio-économiques...). Les entretiens ont été conduits en langue française, en portugais ou en anglais. Ils ont été enregistrés et retranscrits après avoir obtenu le consentement oral des femmes. Les entretiens ont été réalisés suite à l'autorisation des structures dans et avec lesquelles nous avons travaillé, dans des lieux proposés puis choisis par les femmes (chambre de maternité, de néonatologie, domicile des femmes, salle d'attente).

Sociographie du corpus mobilisé :

Dans le corpus ici présenté, toutes les femmes sont arrivées depuis quelques semaines dans la ville dans laquelle nous les avons interrogées, toutes ont migré au cours de leur vie et sont nées à l'étranger, certaines ont eu des grossesses, accouchements, ou autres événements gynécologiques dans leur pays d'origine ou d'autres pays dans lesquels les femmes ont pu résider. Ces différents éléments seront précisés dans les citations au cours du texte ou les vignettes illustratives. Ces femmes sont de différentes nationalités. Elles sont engagées dans des relations de couple hétérosexuelles. Elles sont toutes majeures, résident ou ont un point d'ancrage (même précaire et éphémère) dans les métropoles couvertes par nos enquêtes. Elles peuvent avoir vécu, pour certaines, des issues de grossesses compliquées (enfant de petit poids ou prématuré). Elles ont été rencontrées dans la chambre de leur enfant en néonatologie, ou en maternité, ou dans une salle de consultation ou encore sur leur lieu d'hébergement (appartement; hôtel social; squat). Les femmes peuvent être dans des situations administratives très différentes. Certaines bénéficiaient d'un titre de séjour, d'autres arrivées très récemment n'avaient encore aucune demande de titre de séjour en cours et étaient donc considérées comme "sans papiers", certaines étaient demandeuses d'asile, d'autres déboutées de leur demande d'asile, d'autres dites "dublinées " (dans l'impossibilité d'effectuer une demande d'asile en France, car leur identité avait déjà été relevée dans un autre pays européen où elles étaient passées ou avaient résidé). Elles pouvaient être accompagnées de leur conjoint ayant des statuts tantôt similaires, tantôt différents des leurs ou bien similaires, mais se situant dans des temporalités décalées (pour un couple, le statut de réfugié du conjoint est acquis, celui de la femme est en cours, celui des enfants n'a pas encore été demandé).

\section{La grossesse en migration: les expériences socio-spatiales de}

\section{rapprochement et éloignement}

Le «projet migratoire d'une personne ou d'un couple implique les familles, leur histoire et

leurs relations » (Cattaneo, 2009), et nous proposons ici de centrer notre propos sur la période

\footnotetext{
${ }^{2}$ Développement de l'information statistique pour l'analyse des risques et des inégalités sociales sur le territoire. Etude pluridisciplinaire des facteurs influençant les inégalités sociales de santé (EHESP / 2012-2015).

${ }^{3}$ ANR MIGSAN « Migrations et santé : expériences migratoires et trajectoires de soins pour les migrants primoarrivants en France » (2016-2020) http://migsan.hypotheses.org
} 
de la grossesse. La grossesse en migration, vue par les sciences sociales, implique une approche globale qui interroge la place que cet événement occupe dans une sphère familiale en migration. En situation de migration, la mobilisation d'un réseau de sociabilité revêt un sens particulier, celui de (re) construire des relations sociales (familiales, amicales) pendant l'attente de l'enfant puis en vue de son arrivée, et de son accueil. Elle peut se faire, plus ou moins facilement selon les situations, suivant des réseaux de sociabilité qui se trouvent tantôt à proximité, tantôt multilocalisés. Par exemple, certaines femmes rencontrées ont fait état d'une sociabilité dispersée dans l'espace, avec plusieurs membres de leur famille en migration, depuis plus ou moins longtemps, impliquant des formes de sociabilités à distance des proches lorsque ceux-ci sont dispersés dans différentes villes, différents pays (Encadré 1).

Encadré 1 : Exemple du réseau de sociabilité d'une femme issue d'une famille aux mobilités transnationales Figure 1 :

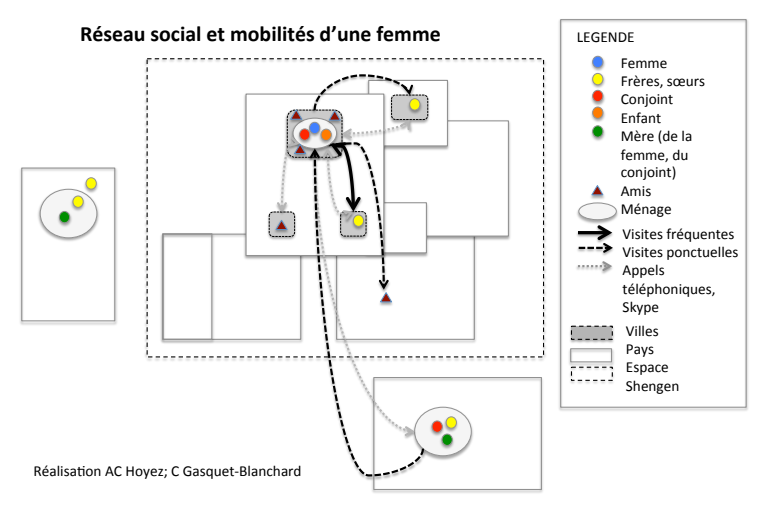

Linda est originaire d'un pays d'Afrique subsaharienne. Elle réside en France avec son conjoint et son enfant. Elle a rencontré différentes personnes dans la ville où elle réside, qui sont devenues des ami.es.

Elle est par ailleurs en lien téléphonique avec une amie qui réside dans une autre ville française et rend visite de manière ponctuelle à une autre amie résidant en Espagne.

La mère de son conjoint habite dans un autre pays avec l'une de ses belles-sœurs et son beau-frère, installés dans une autre ville. Linda échange peu avec sa belle-famille.

Elle est par contre en lien étroit avec l'une de ses sœurs qui vit dans une autre ville française et à qui elle rend régulièrement visite et réciproquement. Pour des raisons principalement économiques, les visites à son frère résidant en Allemagne sont plus ponctuelles, mais les appels sont réguliers, comme ceux avec sa mère et sa sœur qui s'occupent d'un de ses enfants " restés au pays ». Les appels par Skype et téléphone sont réguliers et ils lui rendent visite de manière ponctuelle en France.

La situation de Linda illustre des mobilités et des liens transnationaux tant familiaux qu'amicaux très variés. Les visites aux proches peuvent par exemple se développer auprès de la famille élargie (des parents, de la fratrie, etc.) également en migration. Ces sociabilités sont facilitées par l'expérience commune de la migration, mais s'appuient aussi sur certaines réalités financières (des mobilités moins coûteuses qu'une visite au pays) et administratives (plus de facilité à se déplacer en UE si le titre de séjour français le permet que vers son pays d'origine en raison de la peur de la perte de son visa).

La grossesse vécue à distance de sa famille implique de réinventer les interactions avec les proches (dont principalement la mère, le père, les sœurs et frères, mais aussi les ami.es) quand 
ceux-ci sont restés aux pays. Bien sûr, nous observons le développement de l'usage des outils de communication à distance (internet, téléphone, réseaux sociaux numériques, etc.) (Mattelart, 2009) dans le cadre d'un investissement relationnel auprès de la fratrie et des amitiés créées en migration. Mais le recours aux réseaux sociaux, à l'internet et au téléphone ne compense pas la rencontre physique : il permet surtout de faire perdurer des liens et de gérer l'attente au mieux comme l'illustre cet extrait d'entretien avec Anita, impatiente de savoir si sa mère pourra avoir un visa pour lui rendre visite. Anita est une femme équatorienne, venue pour suivre son mari, chilien. Le couple a le même niveau d'étude, mais elle renonce à chercher un travail pour se concentrer sur sa grossesse, car le couple a rencontré des difficultés pour concevoir cet enfant et parce que son conjoint a décroché un CDD de chercheur post-doctoral dans une université française :

«Parfois [mon mari] ne me comprend pas, la nuit il dort, il ne voit rien, il ne me comprend pas. Quand je suis triste (mon mari dit) le bébé est notre famille, mais moi ma famille me manque, pour moi ma famille c'est ma vie, je vis dans une petite ville, pour moi c'est trop important, lui pense que notre petite famille c'est assez, mais moi j'ai besoin des miens autour de moi. [...] Il est proche de sa famille, mais pas autant que moi, sa famille ils vivent à Santiago, ce n'est pas pareil. Mon frère qui vit en Belgique est venu en février dernier et moi j'y suis allé à Noël! C'est la première fille de la famille, mon père est trop content, car c'est la première petite fille. Ma mère est en train de regarder si elle va pouvoir venir en juillet, j'espère qu'elle va pouvoir venir me voir ».

(Anita, femme équatorienne, sans activité professionnelle, mariée à un Chilien venu en France pour un emploi, enceinte de leur premier enfant.)

Pour les femmes qui rencontrent des difficultés administratives et économiques en France, dans les premiers temps de l'arrivée, la migration se déroule dans un contexte spatial contraint marqué par la peur d'un contrôle de police pour les femmes en situation irrégulière, les difficultés financières qui impliquent de trouver rapidement des ressources dans l'environnement immédiat, la méconnaissance des moyens de circulation dans la ville et l'appréhension de la découverte de l'espace public, le ressenti négatif de l'amoindrissement de leurs mobilités. Tout ceci peut participer à un retard voire un renoncement aux soins ou au suivi de grossesse pour les femmes.

Mais la grossesse en migration peut aussi générer de nouvelles sociabilités qui vont permettre d'agir sur les pratiques spatiales, comme par exemple dans le cadre de rencontres faites dans le milieu professionnel, qui peuvent faciliter et déclencher des mobilités et l'accès à des lieux- 
ressources. C'est le cas de Karen, une femme philippine travaillant comme employée chez une famille française. Elle conduit sa grossesse à distance de son conjoint resté aux Philippines pour gérer son commerce. Son employeuse, accompagne Karen dans ses démarches administratives pour son titre de séjour, son accès aux soins et notamment d'inscription en maternité, dans l'établissement dans lequel elle-même a accouché (et où Karen n'aurait probablement pas accouché si elle avait dû trouver une maternité par ellemême ou par son réseau amical) :

«Yes, yes actually it's my employer who get a rendez-vous here. Yeah, because before she told me, she had two daughters, so the first daughter she gave birth in a private hospital but she didn't told me, and then she told me oh I know a place and then she recommend me here so I told the staff of course and they are very happy of course and it's ah.... yesterday they came here with the two kids. Yeah, so she told me that the hospital is very nice, the staffs are very good so of course you really want to have one like that. So, she, I told her, "okay madame can you help me" and I don't know I'm going to have a house just near here, so it's a good thing for me cos' it's just very near my place, so yeah we took a rendez-vous here last September I think, and then I just moved last December, so it's, yeah it's good for me ».

(Karen, femme philippine travaillant en France ; son mari, philippin, est resté travailler au pays; elle vient d'accoucher de leur premier enfant).

L'information entre femmes sur le suivi de grossesse et tout ce qui en découle : la garde des enfants, la préparation des repas, la compréhension de l'accès aux soins, du suivi de grossesse, ou encore un hébergement provisoire (chez un tiers pendant la grossesse) ou plus durable (l'accès à un logement autonome) se diffuse surtout dans les liens sociaux tissés par les femmes dans la temporalité de leur grossesse.

De fait, nous observons l'évolution, voire le développement, de spatialités très différentes durant le temps de la grossesse, comme cela est décrit par A. Fournant (2009) : 1'espace de la femme enceinte se trouve tantôt restreint, participant à des mobilités contraintes, tantôt s'étend, en induisant une reconfiguration des sociabilités, familiales et amicales multilocalisées.

On constate également l'importance, pour les femmes, d'être en mesure de créer des sociabilités transgénérationnelles et/ou féminines de manière générale autour de l'événement de la grossesse. La principale entrave à la réalisation de mobilités pour ces sociabilités réside dans les aléas liés aux services consulaires pour l'obtention des visas des visiteuses attendues 
(mères, grand-mères : vont-elles avoir leur visa à temps pour pouvoir venir au moment de la naissance ?)

Aussi, la grossesse en migration va déclencher des sociabilités propres à l'accueil de l'enfant et aux solidarités autour de la mère. La grossesse peut être l'occasion de voir se développer autour d'une femme un nouveau réseau social, mais, pour les femmes primo-arrivantes les situations peuvent être très tranchées, notamment au regard des conditions de leur installation dans la durée dans une ville ou un territoire. Les femmes rencontrées ont pu soit rencontrer des difficultés pour créer ou élargir leur cercle de connaissances pour celles qui n'étaient pas stabilisées et autonomes dans leur logement et leur statut administratif; ou soit elles ont pu trouver des ressources supplémentaires quand elles réussissaient à s'appuyer sur leurs ancrages socio-territoriaux multilocalisés.

Enfin, au sein des couples, entre statuts administratifs fluctuants, vie sociale à recomposer autour de la grossesse et de la migration, la vie maritale revêt parfois une dimension spatiale complexe. Certaines femmes rencontrées ont fait état d'une relation de couple transnationale, caractérisée soit par la multiplication des allers-retours (du père) vers Paris ou vers le pays d'origine pour régler des questions de papier dans les consulats ou ambassades, voire l'absence de mobilité d'un père vers sa compagne s'il est resté dans au pays sans autorisation de visa pour venir la rejoindre. Ces situations soulignent à nouveau la présence de cette dialectique d'éloignement/rapprochement provoquée par l'irruption des titres de séjour au sein de la vie de couple, et la façon dont ils mettent en cause la stabilité des couples en obligeant les parents à des mobilités/immobilités contraintes, et ce parfois sur le long terme.

\section{Les couples : gérer la distance ou la proximité}

La littérature scientifique sur la grossesse et l'accouchement met en lumière la reconfiguration des rôles de parents comme révélatrice des normes qui pèsent sur les femmes enceintes (cf. l'image de la bonne mère qui guide la parentalité telle que définie par l'action sociale). Chez les femmes primo-arrivantes en France, cette norme s'accompagne généralement d'une injonction à l'acculturation avec la «culture locale» et pour celles en situation de grande précarité, l'assignation à ces normes de parentalité est encore plus présente. La place des pères est moins étudiée, et nous proposons de nous y arrêter, car, en situation de migration, le père ou conjoint peut endosser des rôles que les femmes (la mère et plus largement les femmes du réseau familial) auraient assuré par ailleurs (Battaglini, 2002). 


\subsection{La place des pères dans les couples en migration}

La littérature scientifique sur la place des pères dans le couple lors des grossesses en migration a montré un surinvestissement ou un nouvel investissement de leur part impliquant une perméabilité de leurs statuts face à la nouveauté du contexte économique, social et à la reconstruction des réseaux sociaux (Battaglini et al., 2002; Jamoulle, 2015). Cette participation vise à pallier le manque de réseau social que pourrait rencontrer leur conjointe, comme le décrivent plusieurs femmes interrogées rencontrées : au sein de ces familles en migration peuvent exister des relations très fortes au sein du couple, particulièrement quand les liens avec les compatriotes ou la famille sont distendus. C'est le cas par exemple, d'un couple algérien hébergé dans la famille du père. Pour eux, l'exigüité des lieux et la promiscuité des liens sont constantes et dégradent les relations familiales : la femme nous explique que l'attitude de son mari, bienveillante envers elle durant sa grossesse (il s'occupe du ménage, de la cuisine, l'emmène en promenade, etc.) tranche avec l'attitude de son beaufrère, ce qui mettra à mal la relation avec les hébergeurs qui les mettront à la porte un peu avant l'accouchement.

D'une autre façon, il est arrivé que la présence des pères durant certains entretiens et leur implication dans la discussion, puisse présager d'un investissement personnel, parfois évoqué par les femmes elles-mêmes : connaissance des termes techniques de la grossesse par le père, de l'état de santé de sa conjointe, volonté d'être présent durant l'accouchement. La plupart du temps, ces investissements personnels proviennent d'un engagement sur le vif, émergeant autant d'un désir individuel que d'une conformation à la normativité médicale et sociale attendue d'eux en France.

En effet, les hommes présents lors des entretiens réalisés (environ 1/3 des situations de l'enquête) se montrent impliqués dans la grossesse de leur conjointe et leur paternité naissante ou reconduite. Ils ont interagi pendant l'entretien en apportant des informations sur la santé de leur femme de manière générale et particulièrement durant la grossesse, ainsi que de celle de leur enfant. Ils ont pu également évoquer les projets de la famille. Leur présence peut être en lien avec un contexte leur permettant d'être présents lors des consultations (arrêt maladie, impossibilité de travailler, ou possibilité de prendre un arrêt de travail) ou une condition pratique mobilisant leur intervention (ce sont souvent eux qui conduisent). Mais celle-ci montre également une mobilisation auprès des enfants.

\section{Encadré 3 :}

L'entretien a lieu avec un couple érythréen en présence de leurs trois enfants. II se déroule en français avec le 
mari et en anglais avec sa conjointe, lui est arrivé en France il y a plusieurs années, et après un parcours de rue et d'errance entre différentes villes françaises. Il est au moment de l'entretien sous protection internationale. Elle est arrivée avec leurs deux filles aînées, il y a un peu moins d'un an, dès la stabilisation du statut administratif légal de son conjoint, sa demande d'asile est en cours. Nous rencontrons le couple en visite postnatale à l'issue de l'accouchement de leur troisième enfant :

- "During the delivery, you were alone or your husband was not really far? You stayed with the girl? "

- « J'ai surveillé les filles. J'ai téléphoné à l’ambulance qui l’a amenée ».

$[\ldots]$

- "Vous ne travaillez pas?».

- « En ce moment, non ».

- "Parce qu'il n'y a pas d'autorisation de travail pour vous ou parce que vous ne trouvez pas?".

- " J'ai l'autorisation, mais en ce moment, je suis un petit peu occupé avec les enfants. J'ai cherché pour dans 3 mois ».

Par ailleurs, le passage à un statut de père en migration apparaît comme un moment sensible : en l'absence du réseau habituel, l'investissement du rôle auprès de l'enfant et l'endossement de la paternité peut prendre de multiples formes : «Les rôles se mêlent, les responsabilités se chevauchent et les parents sont soudainement exposés à l'influence de nouvelles normes sociales, souvent véhiculées par les professionnels de la santé, surtout si les réseaux social et familial sont éclatés » (Battaglini et al., 2002). On passe en quelque sorte à un autre type de pression sociale du désir de conformité, avec néanmoins une répartition des tâches qui persiste entre hommes et femmes. "L’implication des pères représente un facteur de protection en contexte migratoire dans la mesure où ils peuvent combler le vide laissé par le réseau familial» (Battaglini et al., 2002). À la distance spatiale du réseau imposé par la migration, les pères jouent un rôle fondamental dans le bien-être des femmes enceintes et peuvent se retrouver à investir, libres ou contraints, des lieux qui, ne sont pas encore majoritairement investis par les pères dans le suivi de la grossesse et/ou de l'accouchement, comme la salle de naissance ou la salle d'examen gynécologique par exemple (Oakley, 1980).

\subsection{Les couples à l'épreuve des contraintes des normes institutionnelles}

\section{et sociales}

Comme nous l'avons vu, la relation de couple en migration revêt différentes formes. Il existe des couples «à distance » (où les conjoints vivent dans différents pays, ou dans différentes villes dans le même pays). Nous avons rencontré des femmes en situation de monoparentalité, ayant pu laisser un enfant dans leur pays d'origine, après avoir été éconduites ou abandonnées par celui qu'elles appellent généralement «le géniteur». Il existe également des couples séparés longtemps, qui ne se retrouvent que lorsqu'un des deux conjoints se stabilise. Aussi, la dynamique des couples s'inscrit dans plusieurs dimensions sociales et territoriales (Schmoll, 2014) : à plusieurs reprises, nous avons noté que les configurations des 
deux individus formant le couple sont à prendre en compte dans le suivi et le vécu de la grossesse. En effet, le père peut se situer de manière différenciée par rapport au vécu de sa conjointe au regard de son titre de séjour et de l'évolution en cours du contexte institutionnel, administratif et familial.

Dans plusieurs cas, les couples rencontrés ont fait état de leur impossibilité d'être ensemble pour des raisons administratives liées à l'obtention de leurs papiers et se retrouvent séparés, empêchés de se réunir, se retrouver et de vivre ensemble au quotidien. De surcroît, l'absence du père en raison de contraintes administratives, comme nous l'avons évoqué plus haut, peut être lue comme entrant dans l'ensemble des violences faites aux femmes.

Ces séparations longues participent soit à l'éclatement des couples, ou au contraire à les souder encore plus (comme c'est le cas pour le couple érythréen, mentionné plus haut).

Les contraintes administratives ne sont pas uniquement liées aux titres de séjours, mais aussi à la façon dont les services sociaux prennent en charge les femmes. C'est ce que suggère cet exemple d'un couple rom que nous rencontré, arrivé l'un comme l'autre dans le contexte de leurs études et vivant elle dans une métropole de l'ouest (elle) et lui dans une métropole du centre de la France. Suite à l'accouchement, le couple est sans hébergement et on propose à la femme pour sa sortie de maternité une prise en charge en centre maternel. Cette prise en charge dans une «structure mère-enfant», solution généralement proposée en premier par les services sociaux, exclut de fait le père de la solution d'hébergement. Le couple refuse cette proposition. Ce faisant, ils deviennent, aux yeux des soignants et travailleurs sociaux, un couple «non observant» à qui on ne proposera pas d'autre solution dans l'immédiat, en raison de la rareté des places disponibles accueillant des familles.

Pour les femmes qui ont des enfants de différents pères, les tentatives pour regrouper la fratrie sont autant d'épreuves mettant à mal leur statut de mère. C'est le cas d'une femme colombienne, qui, quand nous la rencontrons, vient juste de donner naissance à son $4^{\mathrm{e}}$ enfant. Elle est très anxieuse au sujet de ses deux aînés, pris en charge par l'ASE (leur père, français, est décédé), et de son $3^{\mathrm{e}}$ enfant, dont la garde vient d'être confiée à son $2^{\mathrm{e}}$ mari. Elle est très inquiète aussi pour le devenir de son nourrisson et de son couple : la demande de visa de résident pour son partenaire a été refusée, et il est sur le point de tomber dans la catégorie «sans-papiers ». Aussi, en tant que mère de 3 enfants de nationalité française et d'un enfant pour qui cette nationalité n'est pas acquise et conjointe d'un homme susceptible d'être reconduit à la frontière, elle se trouve prise au piège d'une situation administrative inextricable. Ce contexte de risques et menaces d'origine administratifs la rend anxieuse plus que ses conditions de vie ou de santé personnelles. 
Pouvoir conjuguer vie de couple et vie de famille en situation de migration et au cours de difficultés administratives fait courir à ces femmes le risque d'être catégorisées comme aux marges des normes de la maternité et de la parentalité. Assignées au statut de mère et à devenir sujet (Virole, 2016), ces femmes sont poussées à devoir endosser et à subir les représentations négatives que le monde de l'action sociale et le système de santé véhicule si leurs choix et expériences s'écartent de la norme.

\section{La dynamique familiale en migration : les liens affinitaires entre relation d'aide et de dépendance}

En appui des couples, nous avons pu constater dans les entretiens une mobilisation importante du réseau familial notamment autour de l'organisation de visites à la femme durant sa grossesse, autour de l'accouchement, du soutien après la naissance. La grossesse et la maternité apportent potentiellement un statut à la femme qui lui permet d'acquérir une certaine légitimité dans la famille ou la belle famille. Cet événement implique un changement d'identité sociale pour la femme (qui passe de fille ou belle-fille à mère) visible dans l'organisation dans les lieux de vie pour les couples hébergés, particulièrement si ces familles constituent les tiers hébergeant le couple et ses enfants. C'est le cas par exemple quand les femmes enceintes sont libérées de certaines tâches domestiques (ménage, cuisine, courses). Cependant, ce nouveau statut peut aussi complexifier la relation fille/mère ou belle-fille/bellefamille, notamment quand il y a conflits autour de l'hébergement. Ceux-ci sont fréquemment signalés et peuvent parfois aboutir à la mise à la rue de la femme ou du couple, impliquant des difficultés importantes pour les femmes à l'approche de l'accouchement et/ou à la sortie de la maternité. Cet investissement de la famille et de la belle-famille au moment de la grossesse et de l'arrivée d'un enfant est donc à double tranchant. Par exemple, nous avons pu rencontrer, lors de nos terrains, une femme accompagnée par sa belle-sœur en consultation, cette dernière se faisant traductrice, accompagnatrice et porte-voix de l'histoire reproductive de la femme enceinte qui consulte. Cette figure de la belle-sœur qui investit la grossesse interroge le degré d'autonomie et d'intimité de certaines femmes ou couples au sein du réseau social dans lequel ils évoluent, mais aussi sur le soutien qu'elle peut apporter (on apprendra que la belle-sœur se renseigne pour la femme pour des cours d'apprentissage de la langue française) pas seulement dans l'accompagnement dans le parcours de suivi de grossesse, mais aussi sur l'existence d'un réseau social hors du contexte familial. 
De fait, les spatialités de ces femmes se trouvent très liées à celles du réseau social dont elles disposent et qu'elles établissent, tant dans leurs espaces de socialisation qu'à travers leurs accès aux soins ou à d'autres ressources.

La belle-famille peut être un réseau bienveillant participant à l'insertion de la femme dans la ville (accompagnement à des cours de français, partage d'un carnet d'adresse associatif, aide à se repérer dans l'espace urbain...), mais peut aussi s'avérer participer à maintenir les femmes sans autonomie (traduction par un proche ou présence pour chaque rendez-vous médical, administratif, laissant peu de place à l'expression de la femme). La variabilité des situations décrites par les femmes et observées en consultation nous pousse à nous interroger sur le paradoxe des réseaux familiaux tantôt participant à aider les femmes à acquérir de l'autonomie, tantôt les maintenant dépendantes. Quelles négociations ont lieu au sein des familles, belles familles ou proches hébergeurs? En quoi les expériences des femmes au sein de réseaux de sociabilité familiaux peuvent-elles participer aux choix des femmes pour se loger autrement (certaines préfèreront être dépendantes du 115 que retourner chez leur hébergeur)?

Parmi les femmes que nous avons rencontrées, se trouvent une petite proportion de femmes arrivées en France pour trouver des soins adaptés au fœetus ayant une malformation ou pour faire soigner un enfant qui nécessite des soins qui n'existent pas ou ne sont pas disponibles dans le pays d'origine. L'urgence vitale qui préside à ces mobilités s'accompagne généralement d'un réseau familial transnational mobilisable autour de ces femmes et leurs enfants, dans des projets de long terme liant des problématiques sanitaires d'un enfant à naître.

D'autres situations liant les femmes en migration et leurs familles ont été repérées sur le terrain autour des mères d'enfants restés au pays (confiés à des parents ou des proches). Pour elles, les difficultés vont émerger sur le plan émotionnel et psychologique. La grossesse et la naissance d'un nouvel enfant ravivent un statut de mère qui a dû être mis de côté lors de la migration décidée pour parer aux difficultés qui les ont conduites à partir seules. Les femmes qui peuvent avoir été obligées d'imposer une distance spatiale entre elles et des enfants restés aux pays évoquent toujours un déchirement; en effet, quand il existe des enfants au pays, la naissance apparait comme un moment complexe pour la femme avec des risques accrus de dépression post-partum (Tobin et al., 2018).

La question de la scolarisation des aînés présents en France se pose aussi comme l'illustre la trajectoire de Hawa : 


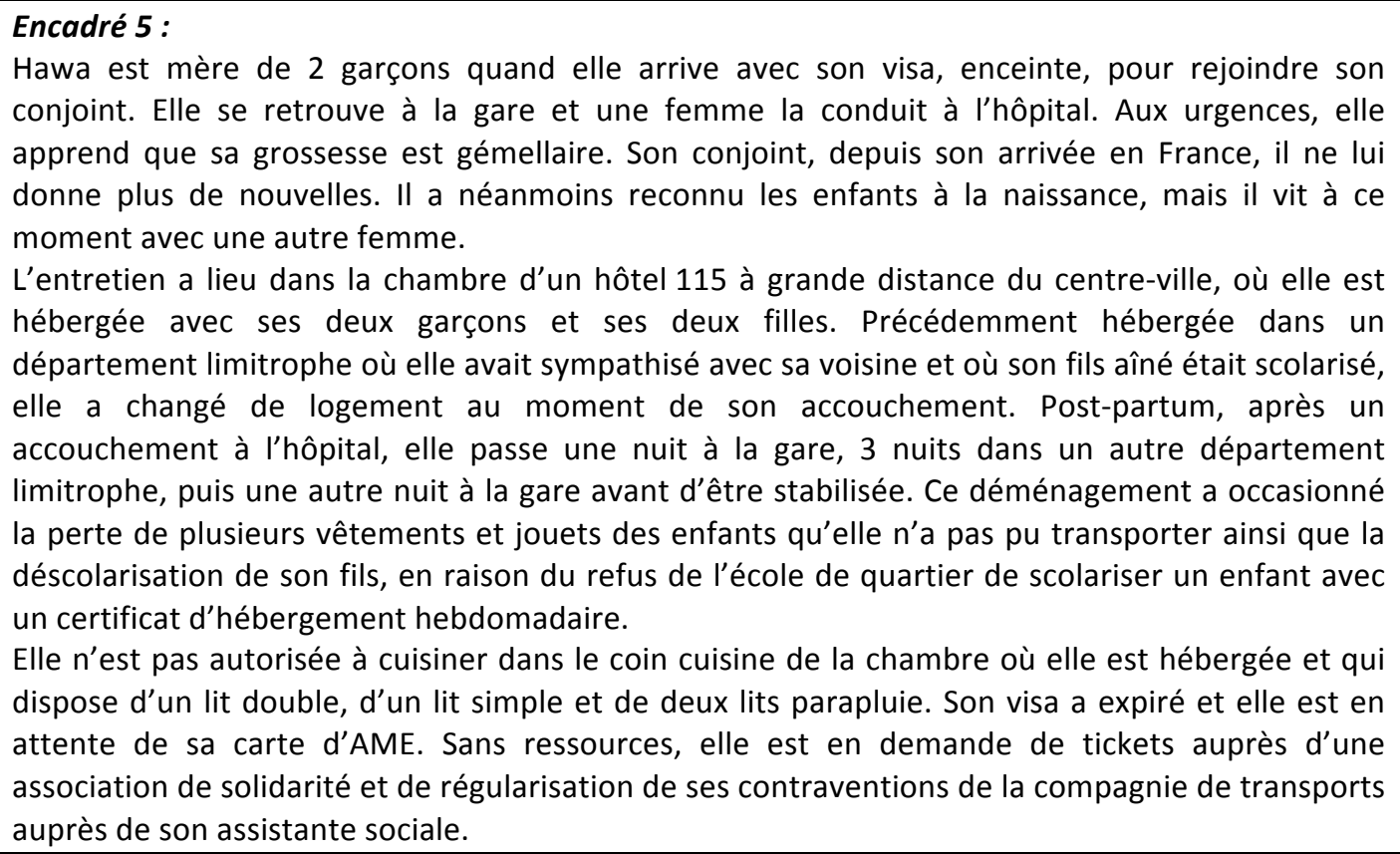

La trajectoire de cette femme et de ses enfants illustre les spatialités contraintes auxquelles peuvent être confrontées les femmes vivant dans un contexte de vie précaire, d'un pays à l'autre, d'un département à l'autre, d'un hôtel à l'autre.

Cela souligne aussi la façon dont la prise en compte de ces conditions de vie participe à mettre à l'épreuve le travail institutionnel, soignant, relationnel qui accompagne les trajectoires de grossesses des femmes.

\section{CONCLUSION}

Pour les femmes dont les entretiens ont été mobilisés dans cette étude on observe une pluralité de situations et de projets : des stratégies liées au travail et à la réussite personnelle, d'autres liées à leur propre protection ou celle de ces proches. Toutes les femmes ne sont pas arrivées en famille, certaines sont arrivées seules, quelques-unes avaient des raisons liées à leur santé ou celle d'un enfant. Pour toutes, elles ont «fait leur vie» en France (ce fut parfois une épreuve), elles ont installé un environnement social qu'elles souhaitent voir perdurer ; elles ont le projet de rester en France. Des conflits avec les conjoints ont été décrits dans les entretiens, donnant fréquemment lieu à des séparations du couple durant la grossesse, renforçant l'isolement des femmes. Par ailleurs, même si un entourage familial peut être présent, celui-ci ne peut pas toujours être mobilisé par les femmes, impliquant pour elles une grande solitude. Si plusieurs situations dramatiques ont été observées, elles ne sont pas la 
norme. Nous avons observé des situations où les primo-arrivantes pouvaient être incluses dans un suivi de soin adapté, se trouver au sein d'un entourage familial bienveillant et vivre des expériences positives.

Autrement dit, aborder la précarité pour les femmes primo-arrivantes avec qui nous nous sommes entretenues ne se résume pas à décrire leurs conditions de vie économiques, sociales et administratives, les conditions de leur accès aux droits de santé et à un logement stable. La prise en considération des aspects affectifs, psychiques peut également entrer en ligne de compte (Davoudian, 2007, 2012; Furtos, 2009; Pinel-Jacquemin et al., 2018) autant que les formes de sociabilité des femmes dans leurs espaces de vie. Ceci plaide pour la prise en compte des dimensions socio-spatiales de la grossesse en migration, tantôt locales, tantôt transnationales, se jouant sur le long terme (la migration et la grossesse comme événement à l'échelle d'une vie) et sur le court terme (l'urgence de se loger, la nécessité de sociabiliser, de faire suivre sa grossesse). En positionnant la grossesse comme événement social, on voit émerger des mobilités individuelles et autonomes chez les femmes enceintes, mais aussi des mobilités accompagnées et/ou suscitées par des proches. On observe des phénomènes sociaux et spatiaux tantôt d'éloignement tantôt de rapprochement selon le réseau relationnel des femmes, leurs ressources, les droits qu'elles peuvent convoquer, l'image que les services sociaux se font d'elles, et ce à différentes échelles.

Cependant, travailler sur ce double événement (la grossesse et la migration) lorsqu'il intervient de manière simultanée implique de situer nos propos pour mieux analyser les effets de la précarité sur les grossesses et ses issues, mieux appréhender ce qui est universel dans la situation de précarité pendant la grossesse et qui se révèle être différent pour les primoarrivantes. 

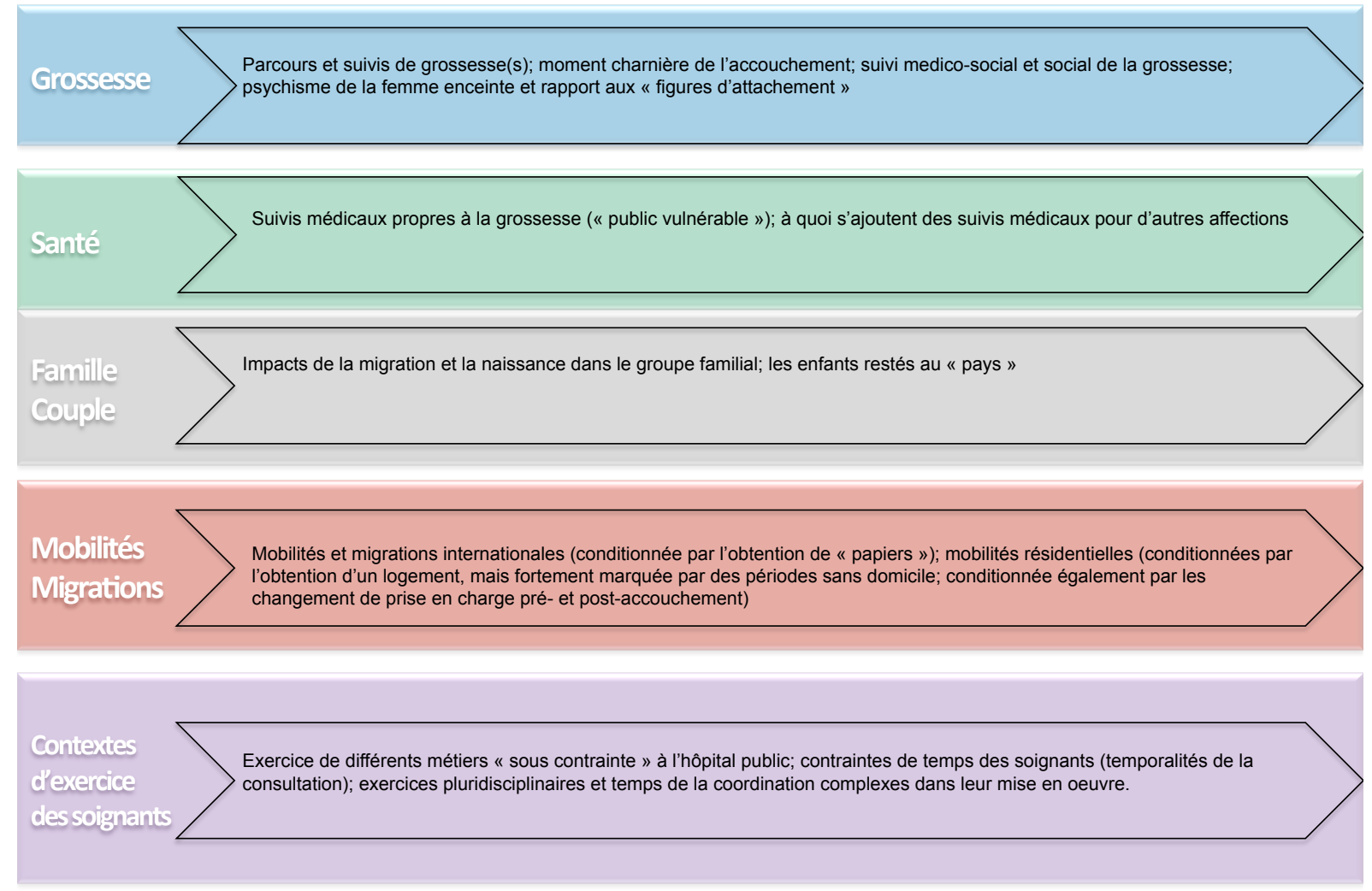

Figure 2 : Articulation des mondes sociaux dans l'approche par trajectoires

La figure 2 propose de prendre en considération l'ensemble des mondes sociaux des femmes enceintes récemment arrivées, autour de leur grossesse, leur santé, de leur situation maritale et matrimoniale, de leur statut migratoire et des contextes dans lesquels elles se trouvent en interaction avec les travailleurs sociaux, les professionnels de santé, ou bien les personnels administratifs. À travers cette figure, nous montrons à la fois l'importance de prendre en compte une approche globale et sur le long terme de la grossesse et de la migration pour mieux comprendre ce qui entre en jeu pour les femmes enceintes en migration à l'échelle du quotidien.

La multiplicité des expériences et des choix de ces femmes montre qu'elles se saisissent de manière habile des situations auxquelles elles sont confrontées, notamment face aux injonctions qui leur sont faites en termes d'attendus sociaux et spatiaux : être hébergée dans tel type de structure si on a tel profil (Planche, 2014), être bénéficiaire de telle couverture sociale si on est détentrice ou non de tel titre de séjour. Ces normes institutionnelles sont érigées en normes sociales, mais sans pour autant correspondre aux attentes et aux besoins des femmes. 


\section{REFERENCES BIBLIOGRAPHIQUES}

Battaglini, A., Gravel, S., Boucheron, L., Fournier, M., Brodeur, J., Poulin, C., DeBlois, S., Durand, D., Lefèbvre, C. \& Heneman, B. (2002). Quand migration et maternité se croisent : perspectives des intervenantes et des mères immigrantes. Service social, 49 (1), 35-69. doi:10.7202/006877ar

Bolzman, Claudio. « Exil et errance », Pensée plurielle, vol. 35, no. 1, 2014, pp. 43-52.

Catarino C., Morokvasic M., 2005, «Femmes, genre, migration et mobilités », Revue européenne des migrations internationales, vol. 21 - $\mathrm{n}^{\circ} 1$ | 2005, 7-27.

Cattaneo M.L., Dal Verme S. (2009). «Conflits familiaux autour de la maternité dans la migration $»$, Dialogue ( ${ }^{\circ}$ 185), p. 79-89.DOI 10.3917/dia.185.0079

Collet, Beate, et Blandine Veith. «Les faits migratoires au prisme de l'approche biographique », Migrations Société, vol. 145, no. 1, 2013, pp. 37-48.

Davoudian, C. (2007). Maternité d'exil. La clinique lacanienne, 12, (1), 69-73. doi:10.3917/cla.012.0069.

Davoudian, C. (2012). Du délit d'existence au déni d'existence : Variations sur les papiers. 1. Dans: C. Davoudian, Mères et bébés sans-papiers: Une nouvelle clinique à l'épreuve de l'errance et de l'invisibilité ? (pp. 79-94). Toulouse, France : ERES.

Duplan K., 2016, Devenir « expat». Performances et pratiques de l'espace du quotidien de femmes en situation de mobilité internationale à Luxembourg. Thèse de doctorat en géographie, sous la direction du Prof. Louis Dupont (Paris IV) et du Dr. Philippe Gerbe (LISER), Université de Paris-Sorbonne, soutenue publiquement à Paris le 13 mai.

Furtos Jean. Demande d'asile et parentalité, un paradigme de la précarité, Cahiers de Rhizome, $\quad \mathrm{n}^{\circ} 37, \quad 2009, \quad$ p. 9-11. $\quad$ http://www.ch-le-vinatier.fr/orsperesamdarra/rhizome/anciens-numeros/rhizome-n37-de-1-exil-a-la-precarite-contemporainedifficile-parentalite/demande-d-asile-et-parentalite-un-paradigme-de-la-precarite-1358.html

Gasquet-Blanchard C., 2020, Solipam et la prise en charge des femmes enceintes dans la rue, ADSP, $n^{\circ} 111$ juin 2020, pp28-9

Gasquet-Blanchard C., Hoyez A-C., 2015, Parcours et trajectoires dans le domaine de la santé. Quelques réflexions issues de l'analyse d'entretiens effectués auprès de femmes migrantes enceintes ou ayant récemment accouché dans la ville de Rennes, in Travaux et Documents $n^{\circ} 39$, Rennes

Gerbier-Aublanc Marjorie, « Des capacités d'agir révélées par le vécu collectif de la maladie? Le cas des femmes dans les associations de lutte contre le VIH », dans : Annabel Desgrées du Loû éd., Parcours. Parcours de vie et santé des Africains immigrés en France. Paris, La Découverte, « Recherches », 2017, p. 243-262. URL : https://www.cairn.info/parcours-de-vieet-sante-des-africains-immigres--9782707196453-page-243.htm

Guerraoui Zohra, Sturm Gesine, «Familles migrantes, familles en changement. Le paradigme de la complexité. L'exemple des familles d'origine maghrébine », Devenir, 2012/4 (Vol. 24), p. 289-299. DOI : 10.3917/dev.124.0289. URL : https://www.cairn.info/revue-devenir-20124-page-289.htm 
Guerry, Linda, et Françoise Thébaud. «Femmes et genre en migration », Clio. Femmes, Genre, Histoire, vol. 51, no. 1, 2020, pp. 19-32.

Jamoulle Pascale, Hommes et pères de milieux populaires. Transformations des paternités en milieux précaires, Cahiers critiques de thérapie familiale et de pratiques de réseaux, 54, 1, 2015, p. 145-163.

Kofman Eléonore, « Genre et migration internationale », Les cahiers du CEDREF, 12 | 2004, 81-97.

Mattelart, T. (2009). Les diasporas à l'heure des technologies de l'information et de la communication : petit état des savoirs. Tic\&société 3 (1-2), 13-57.

Médecins du Monde, 2020. Observatoire de l'accès aux droits et aux soins dans les programmes de Médecins du Monde en France. Rapport de synthèse 2019.

Morokvasic M. (2008). «Femmes et genre dans l'étude des migrations: un regard rétrospectif », Les cahiers du CEDREF, 16, 33-56.

Neyrand Gérard, «Un soutien à la parentalité souvent inadapté aux situations de précarité. L'exemple des foyers monoparentaux », Enfances \& Psy, 2015/3 ( ${ }^{\circ}$ 67), p. 105-112. DOI : 10.3917/ep.067.0105. URL: https://www.cairn.info/revue-enfances-et-psy-2015-3-page105.htm

Oakley, Ann (1980). Women Confined: Towards a Sociology of Childbirth. Londres : Martin Robertson

Pinel-Jacquemin Stéphanie, Koliouli Flora, Kelly-Irving Michelle, « Devenir et être parent en situation de précarité », Bulletin de psychologie, 2018/2 (Numéro 554), p. 593-607. DOI : 10.3917/bupsy.554.0593. URL : https://www.cairn.info/revue-bulletin-de-psychologie-20182-page-593.htm

Planche, Maëlle. «L'errance des mères: du tri entre les situations aux registres de justification des orientations dans un contexte de maternité », Pensée plurielle, vol. 35, no. 1, 2014, pp. 101-112.

Rietch, M-G., 2014, Etude exploratoire quantitative de la base de données du Réseau Solipam, $54 \mathrm{p}$.

Schmoll Camille, 2014. "Gendered spatialities of power in "borderland" Europe. An approach through mobile and immobilized bodies', International Journal of Migration and Border Studies, 1 (2), 173-189.

Tobin C., Di Napoli P., Tatano Beck C. (2018). "Refugee and Immigrant Women's Experience of Postpartum Depression: A Meta-Synthesis ». Journal of transcultural nursing, Vol. 29 (1) 84-100.

Virole-Zajde L., (2016). «Devenir mère, Devenir sujet? Parcours de femmes enceintes sanspapiers en France », Genre, sexualité \& société [En ligne], 16| Automne 2016, mis en ligne le 20 décembre 2016, consulté le 07 octobre 2019. URL : http://journals.openedition.org/gss/3862; DOI : 10.4000/gss.3862 